\title{
Transform Domain Block Based Watermarking using Spatial Frequency and SVD
}

\author{
Sachin Gaur, Vinay Kumar Srivastava
}

\begin{abstract}
Digital image watermarking has been proposed to protect the digital multimedia content. The main objectives of watermarking scheme are robustness, reliability, security against numerous attacks. To improve the imperceptibility, robustness and capacity of the watermarked image, this paper presents a transform domain watermarking method using spatial frequency and block SVD. The spatial frequency is used to select the appropriate blocks for embedding the watermark image by transforming the SVD coefficients of these blocks of the cover image. In this paper first we scramble the cover image by ZIG-ZAG sequencing and then rearranged. After that Shift Invariant Discrete Wavelet Transformed (SIDWT) cover image is partioned in to non-overlapping blocks. Then find out the spatial frequency of these blocks, those blocks which spatial frequency value greater than threshold value are selected for embedding process. Now the watermark image directly embedded by modifying the SVD coefficient of these blocks and get watermarked image. Then inverse process is applied for extracting for watermark image form noisy image. Experimental outcomes show that the proposed scheme is higher imperceptible, robust against various image processing attacks and produce improved results as compared to previous presented schemes
\end{abstract}

Index Terms: Shift Invariant Discrete Wavelet Transform (SIDWT), Singular Value Decomposition (SVD), Spatial Frequency (SF), NCC and PSNR.

\section{INTRODUCTION}

The rapid development of internet and multimedia technology demands security of digital data information over the different communication media. Copyright protection, proof of ownership, unauthorized accessing of information, etc. are the main security concerned related to digital technology. There are several techniques such as watermarking and cryptography to protect the digital information [1-2]. Digital watermarking is one of the suitable methods for intellectual property rights related problems. Watermarking is a process of embedding security features in digital media such as audio, video, image etc. In such a way so that it is imperceptible to others. The process of embedding of watermark should not generate any artifact in image or any other digital media. Watermarking process can create visible as invisible watermarked image. However, due to the various security concerns invisible watermarking process mostly used [3].

Revised Manuscript Received on July 10, 2019.

Sachin Gaur Asst. Professor, Dept. Of Computer Science and Engineering,, Uttarakhand Technical University, BTKIT Dwarahat. (Email:sachingaur1234@gmail.com).

Vinay Kumar Srivastava, Professor, Dept. Of Electronics and Communication Engineering, MNNIT, Allahabad (Email: vinay@ mnnit.ac.in)
The desired features of watermarking are security, capacity, robustness and imperceptibility [4-6].Watermarking algorithm can be broadly classified into two categories' namely spatial domain and frequency domain algorithms. In spatial domain techniques' embedding is done by directly modified the pixel values of the carrier digital data. Spatial domain watermarking is less robust, comparatively less secure and has low complexity [7]. In transform domain techniques such as Discrete wavelet transform, Discrete cosine transform, Singular value decomposition etc. [8-13] extract more information than spatial domain techniques. In these techniques embedding is done by modifying coefficients of transform domain. The transform domain techniques are more robust and have high complexity then spatial domain techniques [7-12].Singular value decomposition (SVD) mostly using transform for watermark embedding by modifying the singular values or $\mathrm{U}$ and $\mathrm{V}, \mathrm{U}$ or $\mathrm{V}$ matrices[11-12].The scaling factor plays an important role for embedding the watermark strength and tradeoff between robustness and imperceptibility. If it has high value than increase the robustness but less imperceptible and vice versa The advantage of block SVD based watermarking is that each block treated individually and improves the robustness of the scheme [14]. The SIDWT-SVD based watermarking overcomes the DWT problems because it is shift invariant and has good spatio frequency localization property [15-20].The spatial frequency refers to the energy content in the image, the image which has sharp edges and lot of detail has high spatial frequency and vice versa [21].In this paper for enhancing the robustness and security, first we scramble the image by ZIG-ZAG sequencing and then rearranged. By using the ZIG-ZAG sequencing the pixel are arranged in a random fashion to increase the robustness. After that SIDWT is applied and segmented the all sub band into non-overlapping blocks and then embedded the watermark in to the cover image. The blocks are selected on the basis of spatial frequency for improving the robustness and imperceptibility.

The rest of the paper systematized as in section II the Literature Survey and briefly explained the SVD, SIDWT and Spatial frequency. Then in section III proposed method for embedding and extracting are presented.

The Experimental results and comparative analysis are shown in section IV. The conclusion of this paper is depicted in section $\mathrm{V}$.

\section{Published By:}




\section{LITERATURE SURVEY}

In this section we summarized the early proposed survey related to DWT-SVD, DCT, block-SVD, entropy, spatial frequency, SIDWT based watermarking methods. Makbol et al. [22] proposed a block based digital image watermarking scheme with DWT-SVD. In this scheme blocks are selected for embedding the watermark on the basis of edge entropy as HVS characteristics and achieved high imperceptibility and robustness. Under et al. [23] presented a block based gray scale logo watermarking scheme. Reddy et al. [24] presented a gray scale watermarking scheme, for embedding watermark image significant block is selected on the basis of the weight factors of wavelet coefficients and enhanced the robustness and security. Bhatnagar et al. [25] proposed a DWT based robust gray scale logo watermarking scheme and for embedding watermark image, the blocks are selected which are arrange ZIG-ZAG sequence and improve the visual imperceptibility, security of the scheme. C.H.Chen et al. [26] proposed a watermarking scheme, in which watermark is embedded in selected block using DCT and improve the visual perceptibility. Frankin et al. [27] proposed entropy based robust watermarking scheme using Hadamard transform techniques for reducing the computation complexity. In this scheme watermark data is embedded in few blocks not all blocks and the applied attacks density is very less even though the imperceptibility and robustness is not so good. Li et al. [28] proposed a new method on selection of image block from source image on the basis of spatial frequency criterion and embedding is done in the selective block to improve the imperceptibility and robustness of the scheme. Liu et al. [21] present a multi focus image fusion techniques for image or videos using DCT. In this scheme block are selected on the basis of spatial frequency for fusion and improve the visual quality of the output image. Rockinger et al. [16] proposed a SIDWT based fusion method for improving the temporal stability and consistency of the scheme. Xin et al. [29] proposed a SIDWT based video fusion method, in this scheme on the basis of entropy the approximation target reason is finds out for fusion the target reason by using SIDWT and improve the results from traditional methods. Ghazy et al. [20] presents a DWT and block SVD based watermarking method. In this scheme watermark image is embedded into all sub bands blocks. In our presented paper for improving the robustness, imperceptibility and security, first we scramble the input image by ZIG-ZAG sequencing and then rearranged. By using the ZIG-ZAG sequencing the pixel are arranged in a random fashion to increase the robustness. After that SIDWT is applied and segmented the all sub band into non overlapping blocks and then blocks are selected on the spatial frequency criteria for embedding watermark image.

\section{A. SVD}

In linear algebra, Singular Value Decomposition (SVD) is numerical tool for analysis the image matrix. It decompose any $n \times n$ image matrix $M$ into three matrices $G_{s} H$ and $K$ as $M=G H K^{T}$, where $G$ and $K$ are the left and right singular orthogonal matrices, $H=\operatorname{diag}\left(\mu_{i}\right)$ is a diagonal matrix known as singular matrix, where $\mu_{i}, i=1,2,3 \ldots$ nis singular values. SVD is using in various applications such as image compression, image hiding, image watermarking, and noise reduction [12,30].The left and right orthogonal matrices possessed the geometric information of the image. $G H$, together known as principal components. The singular values hold the intensity information of the image. The SVD can be applied on square as well as rectangular image also. It can be defined as:

$$
\begin{aligned}
M & =G H K^{T}(1) \\
& =\left[g_{1}, g_{2}, \ldots, g_{n}\right] \times\left(\begin{array}{cccc}
\mu_{1} & 0 & \ldots & 0 \\
0 & \mu_{2} & \ldots & 0 \\
\vdots & 0 & \cdots & 0 \\
0 & 0 & \ldots & \mu_{n}
\end{array}\right) \times\left[k_{1}, k_{2}, \ldots k_{n}\right] \\
& =\sum_{i=1}^{r} \mu_{i} g_{i} k_{i}
\end{aligned}
$$

Where ' $r$ ' signifies the rank of matrix ${ }^{M}$. SVD has intrinsic property and the singular values contains the consistency properties that is minor alteration in the singular values do not affect the visual property of the image.

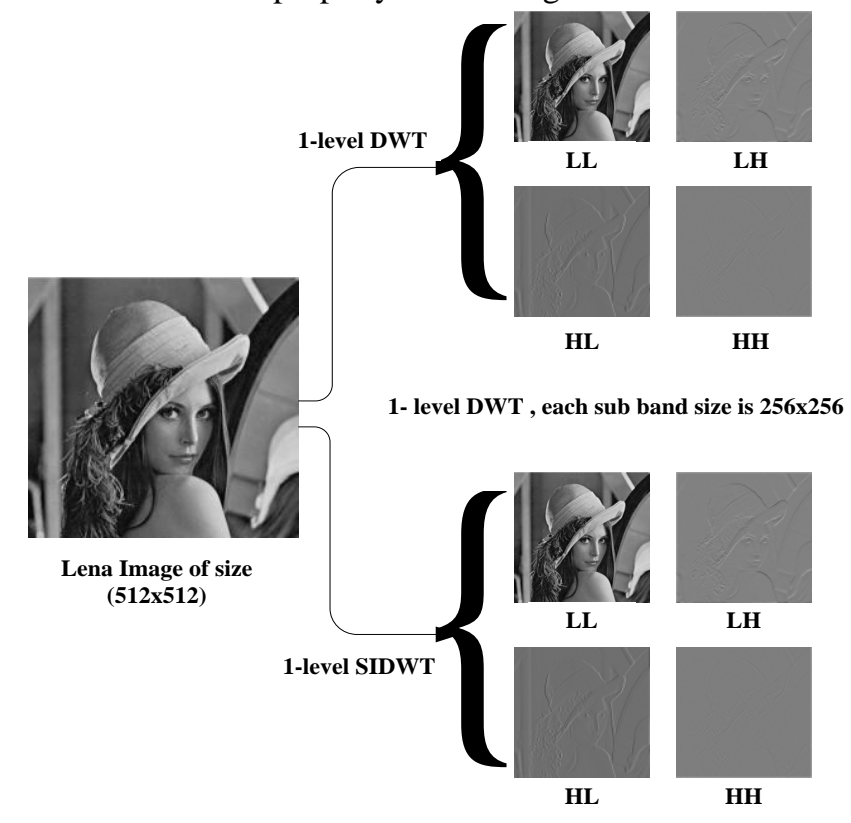

1- level SIDWT , each sub band size is $512 \times 512$

Fig.1. 1-level DWT and SIDWT sub band decomposition (Lena B. SIDWT image of size $(512 \times 512)$

The Shift Invariant Discrete Wavelet transform (SIDWT) is presented by Rockinger et al. [16]. In various literature survey the SIDWT known as different nomenclature as wavelet translation DWT, Shift invariant frames, Maximum overlap Discrete wavelet transform etc. [17-19]. Due to shift variant property in DWT, the maximum variation occurs in the DWT coefficients and the result is inaccurate extraction of the multimedia data. This drawback of DWT can be flash out in SIDWT because it is shift invariant. This leads to extract reconstruction of image. SIDWT produces the same size of sub bands as the original image as shown in Fig.1. 
The size of the approximation and details sub bands after applying SIDWT is same as the original image. The SIDWT discard the down sampling over complete wavelet coefficients. The Fig.1 shows the sub bands images after applying 1-level DWT and SIDWT respectively. The size of sub bands at each level decrease in DWT but same in SIDWT.
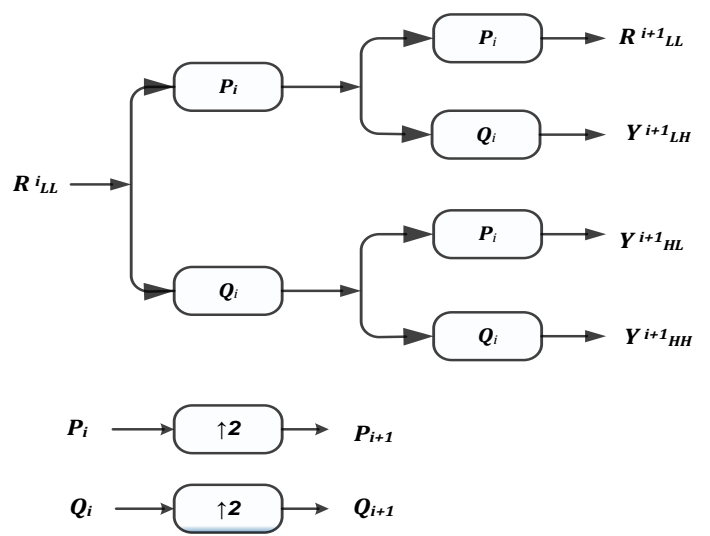

if 2 , shows the up - sampling by a factor 2

\section{Fig.2. 1-level decomposition of SIDWT}

Fig.2. shows the row wise and column vise decomposition after applying SIDWT and each row is decompose in ${ }^{\prime} P$ ' and ${ }^{I} Q^{t}$, further row is then decompose in to column vise and get sub bands at 1-level decomposition. $Y_{L H}^{i+1}, Y_{H L}^{i+1}$ and $Y_{H H}^{i+1}$ represent the detail sub bands. $R_{L L}^{i+1}$ is the approximation low frequency sub band and this divided in to next level. At each level the coefficients is modified by up sampling by a factor $2^{(i-1)}$ in $i^{\text {th }}$ level. The advantages are enhancing the temporal stability, consistency and also SIDWT based digital image processing methods is highly robust than DWT based techniques [20].

The SIDWT can be obtained by using the following equations from (equation 2-7). If $A_{i}(n)$ is the wavelet coefficient and the scaling sequence is $l_{i}(n)$ then equation can be written as.

$$
\begin{aligned}
& A_{i+1}(n)=\sum_{k} m\left(2^{i} \cdot k\right) \cdot l_{i}(n-k)(2) \\
& l_{i+1}(n)=\sum_{k} d\left(2^{i} \cdot k\right) \cdot l_{i}(n-k)
\end{aligned}
$$

For incremented next decomposition level ${ }^{l_{i}(n)}$ considered as an input signal. $m\left(2^{i} \times k\right)$ and $d\left(2^{i} \times k\right)$ is the low pass and high pass filter respectively. $D(z)$ and $M(z)$ are the ztransform of the $d(k)$ and $m(k)$.

$$
\begin{aligned}
& D(z) \cdot D\left(z^{-1}\right)+D(z) \cdot H\left(-z^{-1}\right)(4) \\
& M(z)=z H\left(-z^{-1}\right)(5)
\end{aligned}
$$

When, SIDWT applied on the input image ${ }^{\Psi(x, y)}$. $\Psi(x, y) \rightarrow \operatorname{SIDWT} \rightarrow \varphi_{\left(k_{1}, k_{2}, j\right)(6)}$

Where $\tilde{\Psi}\left(k_{1}, k_{2}, j\right)$ represents the wavelet coefficient. If $(\Delta x, \Delta y) \in R$ are the shift in the input image. Due to the shift

invariant the output coefficient exactly same as input coefficient represents as:

$$
\Psi(x+\Delta x, y+\Delta y) \rightarrow \operatorname{SIDWT} \rightarrow \Psi\left(k_{1}^{s}, k_{2}^{s}, j\right)(7)
$$

Where $k_{1}^{s}=k_{1}+b_{1} . \Delta x$ and $k_{2}^{s}=k_{2}+b_{2} \cdot \Delta y$ for some $\left(b_{1}, b_{2}\right) \in R$. If the coefficient exactly the same then $b_{1}=b_{2}=0$.

\section{Spatial Frequency $(\mathrm{SF})$}

Spatial frequency means the extent of detail present in a scene per degree of visible perspective. An Image with sharp edges and less details have high spatial frequency than one compared of large coarse edges [21, 28]. It is rectified version of energy measure using image gradient. The spatial frequency shown hear can be described by the following equations.

$$
S F=\sqrt{(P F)^{2}+(D F)^{2}}(8)
$$

Where ${ }^{P F}$ and $D F$ are the row frequency and Column frequency respectively and can be described as:

$$
\begin{aligned}
& P F=\sqrt{\frac{1}{N \times N} \sum_{a=1}^{N-1} \sum_{b=2}^{N}\left\{\Upsilon(a, b)-Y(a, b-1\}^{2}(9)\right.} \\
& D F=\sqrt{\frac{1}{N \times N} \sum_{a=2}^{N} \sum_{b=1}^{N-1}\{Y(a, b)-Y(a-1, b)\}^{2}(10)}
\end{aligned}
$$

$\mathrm{N}$, is the size of the blocks or image

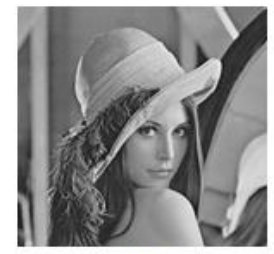

(a)

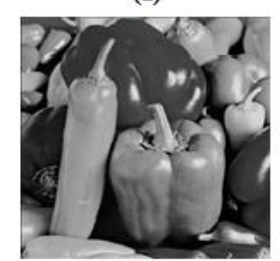

(d)

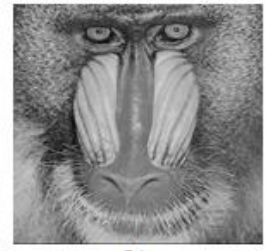

(b)

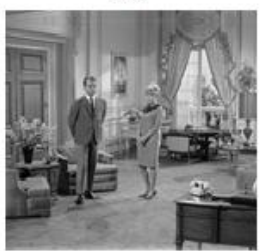

(e)

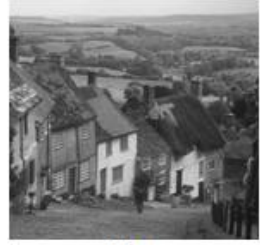

(c)

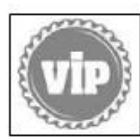

(f)

Fig.3. Cover Images (a) Lena (b) Baboon (c) Goldhill (d) Peppers (e) Couples of size $(512 \times 512)$ and Watermark image (f) VIP logo of size $(128 \times 128)$ 


\section{PROPOSED SCHEME}

In this section presents a digital image watermarking algorithms that embeds a watermark image into threshold determined spatial frequency selected blocks of ZIG-ZAG rearranged SIDWT transformed cover image by transforming the SVD coefficients of these block. The embedding and extracting block diagram are shown in Fig.4., Fig.6. and the algorithm steps are given as:

(a) Watermark Embedding steps:

1. Reordering of cover image ' $I$, using ZIG-ZAG sequencing and rearranged then get cover image ' $I_{R}$ '.

2. Apply SIDWT on ' $I_{R}$ ' image and then segmented into $n \times n$ non-overlapping blocks of all sub bands.

3. Calculate the spatial frequency of every block of all sub bands by using the given equation 9-10.

4. Now find out the spatial frequency each block and select those blocks which have spatial frequency more than threshold value for embedding the watermark image.

5. Apply SVD on selected blocks of all sub bands

$$
X^{i \theta}=U^{i \theta} S^{i \theta} V^{i \theta^{T}}(11)
$$

Where $\mathbb{l}^{\text {th }}$ is selective block and $\theta=\{L L, L H, H L$ and $H H$ sub bands

6. Modifying the singular values of selected blocks by directed embedding the watermark image and then applies SVD.

$$
S^{i \theta}+\alpha W=U_{W}^{i \theta} S_{W}^{i \theta} V_{W}^{i \theta^{T}}(12)
$$

7. Now apply inverse SVD such as:

$$
P^{i \theta}=U^{i \theta} S_{W}^{i \theta} V^{i \theta^{T}}(13)
$$

8. Apply inverse SIDWT to obtain watermarked image 9.Apply inverse ZIG-ZAG process and rearranged then get watermarked image.

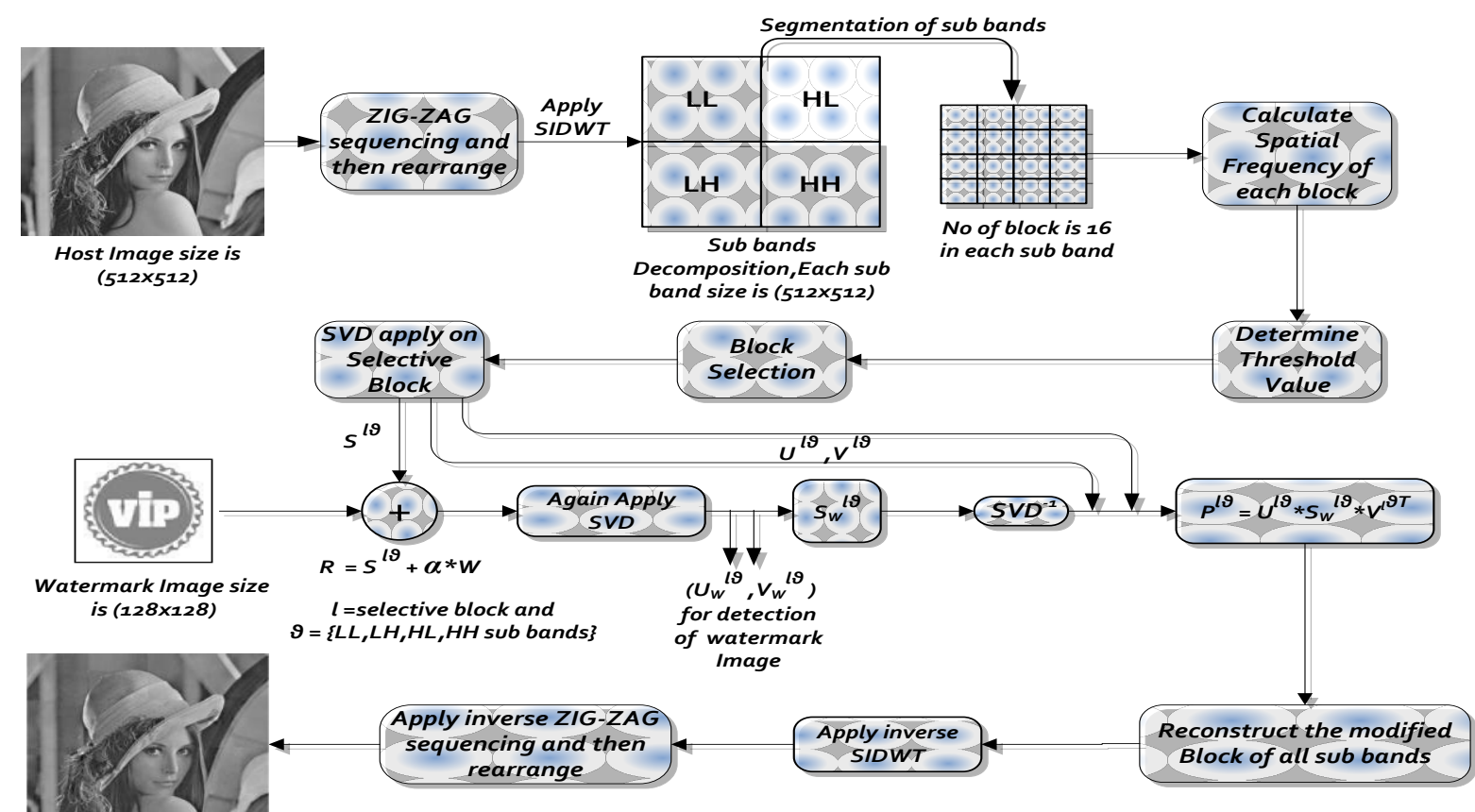

Watermarked Image size is $(512 \times 512)$

Fig.4. Watermark Embedding Block

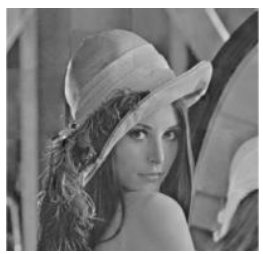

(a)

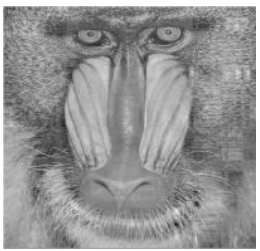

(b)

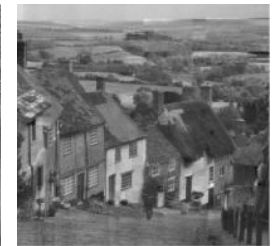

(c)

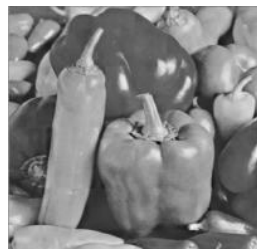

(d)

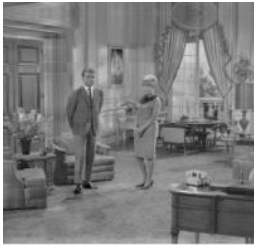

(e)

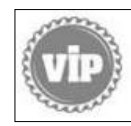

(f)

Fig.5. Watermarked images (a) Lena (b) Baboon (c) Goldhill (d) Peppers (e) Couples of size (512 $\times 512)$ and Extracted Watermark image (f) VIP logo of size $(128 \times 128)$ 
(b) Watermark Extracting steps:

1. Apply ZIG-ZAG scanning process and rearranged the watermarked image $W^{*}$ and get the image $W^{* *}$.

2. Apply SIDWT on rearranged image $W^{\text {s? }}$.

3. Break the image $W^{* 3}$ into $n \times n$ non overlapping block of all sub bands.

4. Now calculate the spatial frequency of each block and that block which have spatial frequency more than threshold value are selected.
5. Apply SVD on these selective frequency blocks such as:

$$
P^{i \theta *}=U^{i \theta *} S_{W}^{i \theta *} V^{i \theta * *^{T}}(14)
$$

6. Now applying inverse SVD on selected blocks as:

$$
P^{i \theta * *}=U_{W}^{i \theta} S_{W}^{i \theta *} V_{W}^{i \theta}(15)
$$

7. Applying this operation and get the extracted watermark image $W E^{*}$ as.

$W E^{*}=\frac{P^{i \theta *}-S^{i \theta}}{\alpha}(16)$

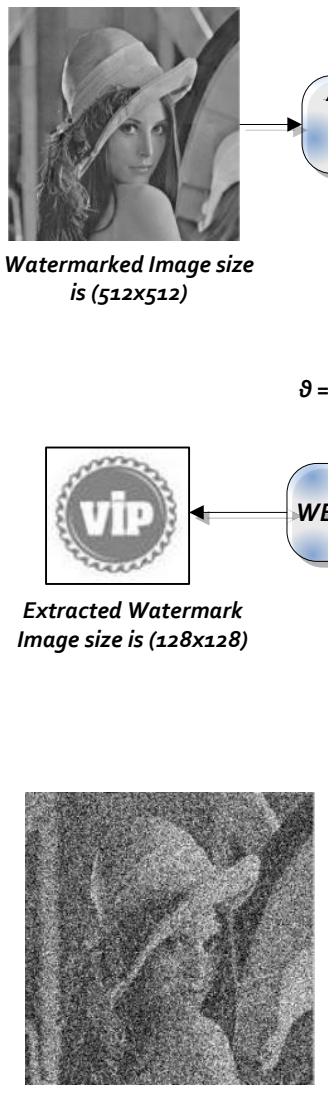

(a)

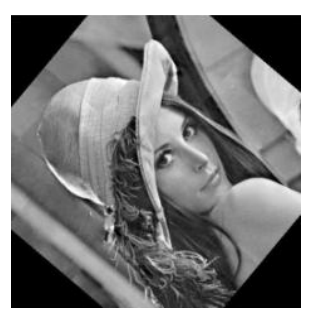

(e)

Fig.6. Watermark Extraction Block Diagram

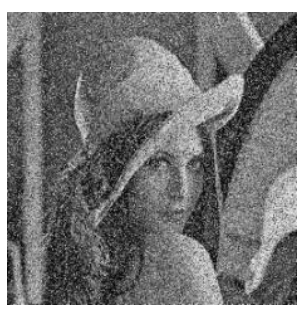

(b)

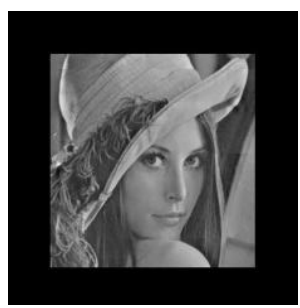

(f)

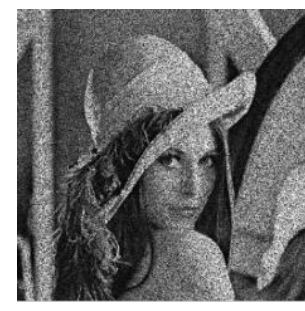

(c)

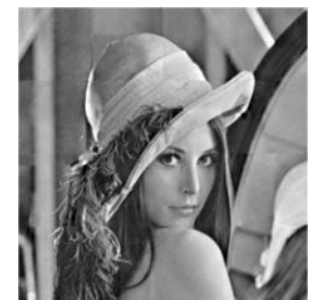

(g)

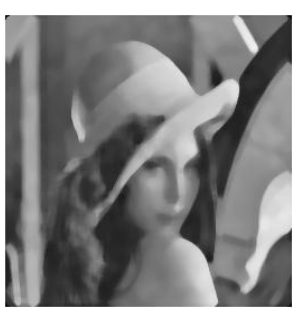

(d)

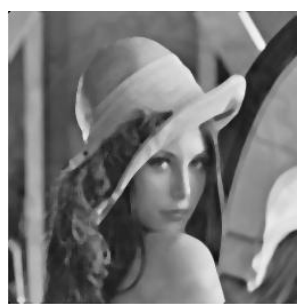

(h)

Fig.7. Watermarked Images After applying attacks (a) Gaussian Noise (M=0,V=0.5) (b) Salt and Pepper Noise (0.4) (c) Speckle Noise(0.4) (d) Median filtering (7×7) (e) Rotation

(f) Cropping (g) Resizing (512-256-512) (h) Compression

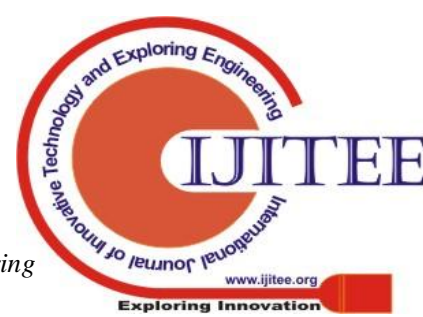




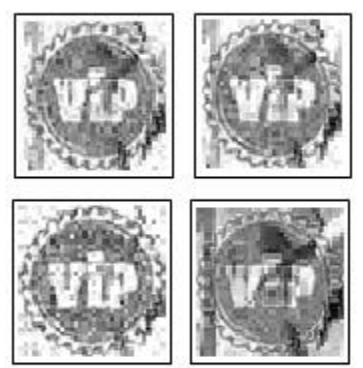

(a)
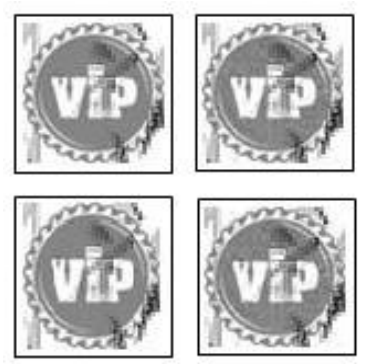

(c)
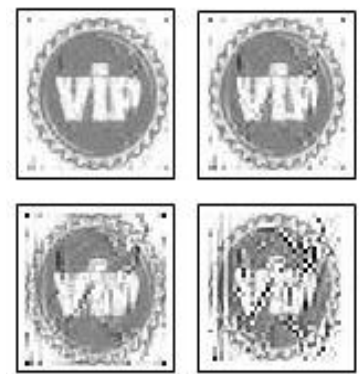

(e)
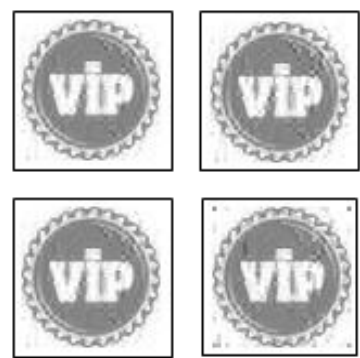

(g)

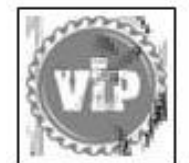

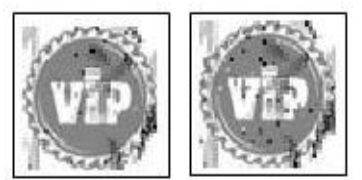

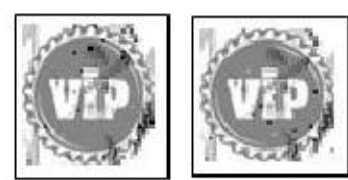

(b)
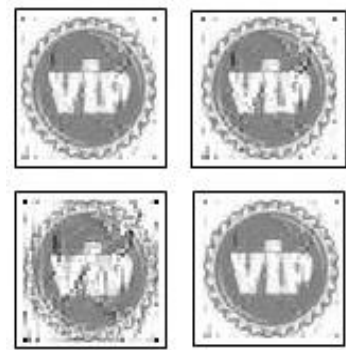

(d)
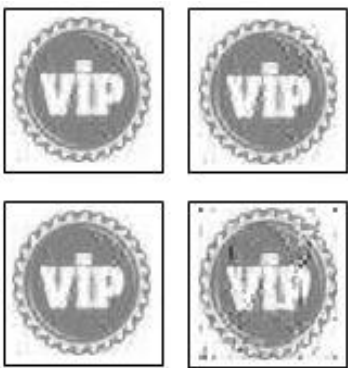

(f)
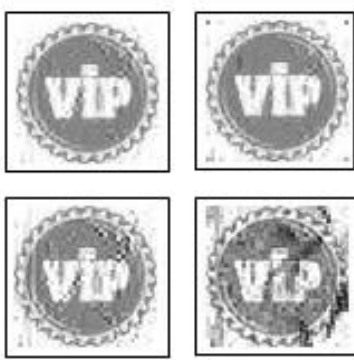

(h)
Fig.8. Extracted Watermark Images After applying attacks (a) Gaussian Noise $(\mathrm{M}=0, \mathrm{~V}=\mathbf{0 . 5})$ (b) Salt and Pepper Noise (0.4) (c) Speckle Noise (0.4) (d) Median filtering $(7 \times 7)$ (e) Rotation (f) Cropping (g) Resizing (512-256-512) (h) Compression

Table.1 Imperceptibility and Robustness measures between different cover images, watermarked image and extracted watermark, original watermark.

\begin{tabular}{|l|l|l|l|l|l|}
\hline \multirow{3}{*}{$\begin{array}{l}\text { PSNR/ } \\
\text { NCC }\end{array}$} & \multicolumn{6}{l}{$\begin{array}{l}\text { Imperceptibility and Robustness Measures } \\
\text { (without attacks) }\end{array}$} \\
\cline { 2 - 6 } & Images \\
\cline { 2 - 6 } & Lena & Baboon & Peppers & Boat & Goldhill \\
\hline PSNR & 60.744 & 58.347 & 57.918 & 58.886 & 54.375 \\
\hline NCC & 0.9993 & 0.9981 & 0.9873 & 0.9885 & 0.9789 \\
\hline
\end{tabular}

Table.2 NCC values between extracted watermark and original watermark after applying attacks with different images

\begin{tabular}{|l|l|l|l|l|l|}
\hline \multirow{2}{*}{ Attacks } & \multicolumn{4}{|l|}{ NCC Values } \\
\cline { 2 - 6 } & \multicolumn{4}{|l|}{ Images } \\
\cline { 2 - 6 } & Lena & Baboon & Goldhill & Peppers & Couples \\
\hline $\begin{array}{l}\text { (a) Gaussian } \\
\text { Noise(M=0,V=0.5) }\end{array}$ & 0.7718 & 0.7575 & 0.7078 & 0.7589 & 0.7118 \\
\hline $\begin{array}{l}\text { (b) Salt \& Pepper } \\
\text { noise(0.4) }\end{array}$ & 0.8571 & 0.8057 & 0.8415 & 0.8187 & 0.7991 \\
\hline (c) Speckle noise(0.4) & 0.8478 & 0.7939 & 0.8129 & 0.8292 & 0.7559 \\
\hline $\begin{array}{l}\text { (d) Median } \\
\text { filtering(7×7) }\end{array}$ & 0.8381 & 0.8083 & 0.7899 & 0.7932 & 0.8015 \\
\hline (e) Rotation(50) & 0.5738 & 0.5249 & 0.5153 & 0.5058 & 0.4513 \\
\hline (f) Cropping (30\%) & 0.6989 & 0.6712 & 0.6771 & 0.6953 & 0.5987 \\
\hline $\begin{array}{l}\text { (g) } \\
\text { Resizing(512-256-512 } \\
\text { ) }\end{array}$ & 0.8998 & 0.8188 & 0.8385 & 0.8088 & 0.7877 \\
\hline (h) Compression & 0.8173 & 0.7749 & 0.7221 & 0.8011 & 0.7876 \\
\hline
\end{tabular}

\section{EXPERIMENTAL RESULTS}

The performance of the presented spatial frequency based watermarking scheme can be calculated by using MATLAB15. This scheme applied on different standard images shown in Fig.5. The size of the cover image is $512 \times 512$ and the watermark image is $128 \times 128$ and the image is containing 64 blocks. Now we calculate the spatial frequency by using the equation. The blocks which has maximum spatial frequency then threshold value has maximum energy are consider for watermark embedding. The visual analysis can be calculated by finding out the Peak Signal to Noise Ratio (PSNR) by the given equation (18). And the robustness can be calculated by finding out by using the given equation (19). The accepted value of PSNR is $30 \mathrm{~dB}$ and the range of NCC is between -1 to +1 and its acceptable value is 0.75 .

The PSNR value of cover image Lena is 60.744 and the correlation coefficients value without attack between extracted watermark and original watermark is 0.9993.In this paper the comparative result are shown in Fig.9-12 and Table.1-4 by taking Lena as cover image and VIP logo as a watermark image.

The PSNR can be finding by the given formula as.

$$
\text { PSNR }=10 \log _{10}\left[\frac{\max (x(i, j))^{2}}{M S E}\right](18)
$$

MSE (Mean square error between cover image $x$ and watermarked image $y$ ) can be calculated as

$$
\text { MSE }=\frac{1}{m \times n} \sum_{i=1}^{M} \sum_{j=1}^{N}\left[x\left(i_{i} j\right)-y\left(i_{i} j\right)\right]^{2}(19)
$$

For robustness we can calculate the normalize correlation coefficient (NCC) by given formula.

$$
N C(w, \bar{w})=\frac{\sum_{i=1}^{M} \sum_{j=1}^{W}\left[w(i, j)-\mu_{W}\right]\left[\bar{w}(i, j)-\mu_{\mathbb{W}}\right]}{\sqrt{\sum_{i=1}^{M} \sum_{j=1}^{M}\left[w(i, j)-\mu_{W}\right]^{2}} \sqrt{\sum_{i=1}^{M} \sum_{j=1}^{N}\left[\bar{w}(i, j)-\mu_{W}\right]^{2}}}(20)
$$

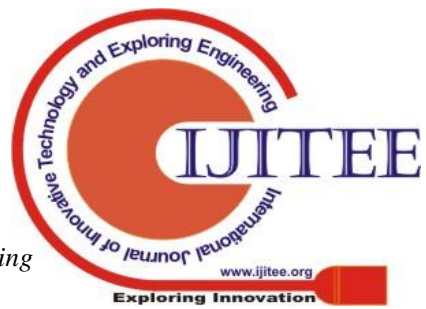


International Journal of Innovative Technology and Exploring Engineering (IJITEE)

ISSN: 2278-3075, Volume-8, Issue-9S4, July 2019

Table.3 Comparison between Previous existing scheme and Proposed Methods

\begin{tabular}{|c|c|c|c|}
\hline \multicolumn{4}{|c|}{ Detail Comparison } \\
\hline & Ghazy et al.[20] & Bhatnagar et al.[25] & Proposed Scheme \\
\hline Type of operating Transform & DWT+SVD & DWT & SIDWT+SVD \\
\hline Size of Cover Image & $256 \times 256$ gray & $256 \times 256$ gray & $512 \times 512$ gray \\
\hline Type of Method & Non- Blind & Non- Blind & Non- Blind \\
\hline $\begin{array}{l}\text { Operation on Watermark } \\
\text { before Embedding }\end{array}$ & $\begin{array}{l}\text { Apply SVD, then embedding into } \\
\text { all blocks }\end{array}$ & $\begin{array}{l}\text { No operation, Direct embedding } \\
\text { into selective blocks }\end{array}$ & $\begin{array}{l}\text { Apply SVD, then Direct embedding into } \\
\text { selective blocks }\end{array}$ \\
\hline Block Selection Criteria & No Criteria, Selects all Blocks & On the basis of Variance & On the basis of Spatial Frequency \\
\hline Addition of Noise & Up to $10 \%$ & Up to $40 \%$ & Up to $50 \%$ \\
\hline Embedding Sub bands & All sub bands & All sub bands & All sub bands \\
\hline Median Filtering & Up to $3 \times 3$ & Up to $10 \times 10$ & Up to $11 \times 11$ \\
\hline Embedding Quality & Loosy & Loosy & Loosy \\
\hline Rotation & Up to $15^{\circ}$ & Up to $40^{\circ}$ & Up to $50^{\circ}$ \\
\hline Size of the Watermark & $16 \times 16$ & $32 \times 32$ & $128 \times 128$ \\
\hline Scaling Factor & $\begin{array}{l}0.05 \text { for LL and } 0.005 \text { for } \\
\text { LH,HL,HH sub bands }\end{array}$ & $\begin{array}{l}0.45 \text { for LL sub band and } 1.0 \text { for } \\
\text { LH,HL,HH sub bands }\end{array}$ & $\begin{array}{l}0.3 \text { for LL sub band and } 0.6 \text { for LH,HL,HH } \\
\text { sub bands }\end{array}$ \\
\hline Type of watermark & Binary & Gray scale & Gray Scale \\
\hline Tested Input Image & Cameraman & Lena & Lena, Baboon, Goldhil, Peppers, Couples \\
\hline
\end{tabular}

Table. 1 Show the PSNR values by taking different original images(Lena, Baboon, Peppers, Boat, Couples) and watermarked images as well as also shows the NCC values between Original watermark(VIP logo) and extracted watermark with no attacks. Table. 2 presents the maximum correlation coefficient value between original watermark and extracted watermark after applying different attacks (Gaussian noise, speckle noise, salt and pepper noise, median filtering, rotation cropping, resizing, and compression).Table. 3 shows the detail comparison between previous existing scheme and presented method.Table.4shows the imperceptibility comparison between proposed and previous existing scheme.

Table.4 Imperceptibility comparison between our and previous presented scheme

\begin{tabular}{|c|c|}
\hline Schemes & PSNR \\
\hline Proposed Scheme & 61.837 \\
\hline Ghazy et al.[20] & 46.386 \\
\hline Bhatnagar et al.[25] & 57.746 \\
\hline
\end{tabular}

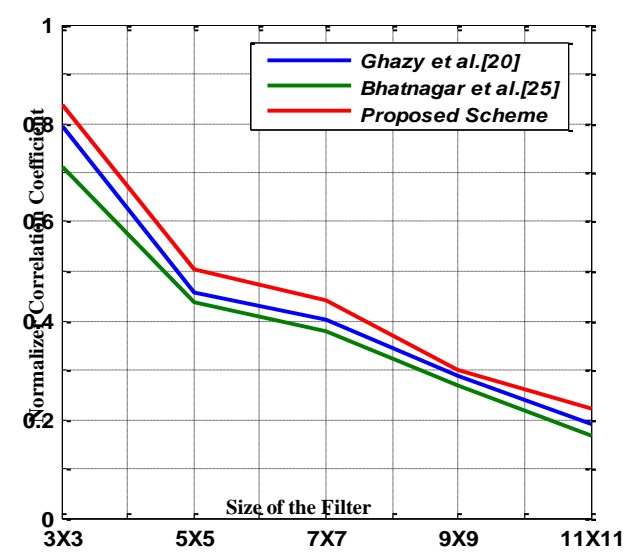

Fig.9 Response of the Median filtering Attack

Fig.9, the graph shows the median filtering attack response with different filter size in between previous presented schemes
Ghazy et al.[20], Bhatnagar et al.[25] and our proposed scheme.

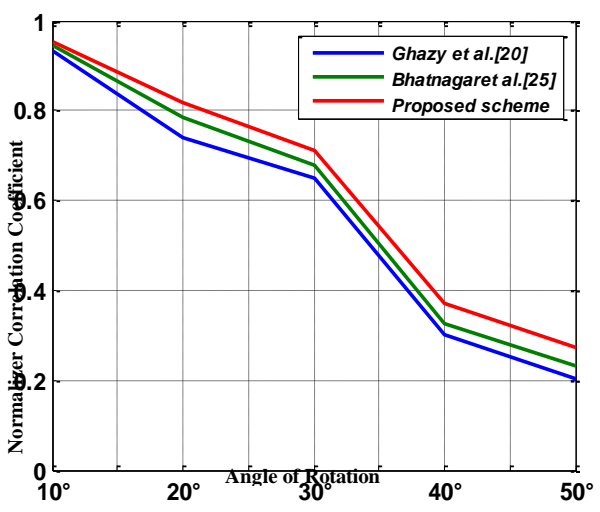

Fig.10 Response of the Rotation attack

Fig.10 shows the rotation attack response with different angle in between previous presented method and our presented method. The graph shows that the proposed method gave the better response then previous scheme.

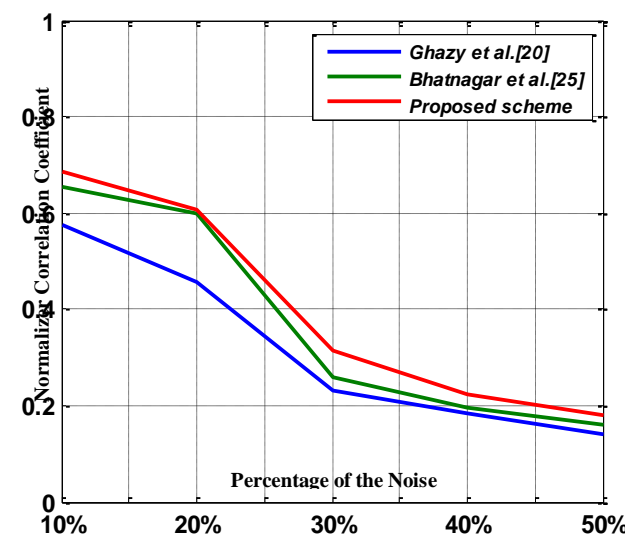

Fig.11 Response of the Gaussian Noise attack

Published By: 
Fig.11 shows the response of the Gaussian noise attack with different percentage of noise in between previous presented scheme and our proposed scheme. Seeing this graph we can say that the proposed scheme gave the better response then previous schemes.

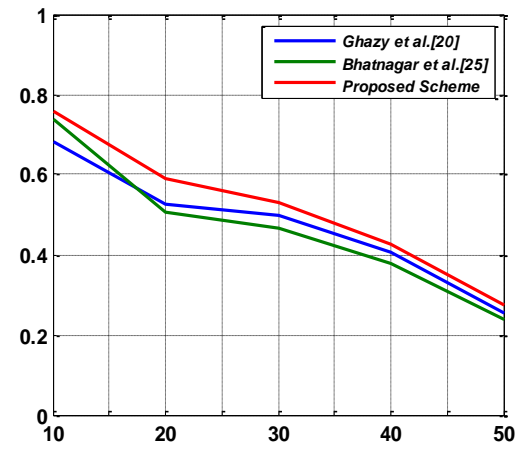

Fig.12 Response of the Compression attack

Fig.12 shows the response of compression attack with different compression ratio in between previous presented scheme and our proposed scheme. After analysis these numerical values in different tables from Table.1-4 and graphs from Fig.9-12, we can say that the presented scheme is resist against different attacks such as Gaussian noise, speckle noise, Pepper and salt noise, median filtering, rotation, compression and have high capacity and more imperceptible and highly robust.

\section{Comparative analysis}

In this section the proposed scheme compared with the previous presented scheme. Ghazy et al. [20] and Bhatnagar et al. [25] In comparison the cover host image size is $512 \times 512$ and watermark image size is $128 \times 128$. Ghazy proposed a DWT-SVD based block wise digital image watermarking scheme, in this proposed scheme the host image size is $256 \times 256$ and watermark image size is $16 \times 16$, and modify the singular value of all blocks of host image for embedded. In this scheme watermark is embedded into all blocks, due to this increase the payload and reduce the imperceptibility. Bhatnagar et al. proposed a DWT based robust logo watermarking scheme, in this scheme each blocks are arranged via ZIG-ZAG sequence, after that calculate the variance of each block determine the threshold value and then find the selected blocks, then watermark is embedded into selected blocks not all blocks so reduce the payload and increase the imperceptibility. In our proposed scheme for improving the imperceptibility, robustness and capacity of the watermarked image, SIDWT transformed cover image is portioned in to non- overlapping blocks and after that select those block which has spatial frequency value greater than the threshold value and then watermark image is embedded in to selected blocks. After seeing the numerical values from Table.1-4 and analysis the graph shown in Fig.9-12 conclude that the presented scheme is more imperceptible, highly robust, low data payload and perform better than previous presented schemes.

\section{CONCLUSION}

This paper presents a new approach of watermarking in the transformed domain using spatial frequency and block SVD method. For improving the robustness, the image pixels are reordered by using ZIG-ZAG scanning. The watermark image is embedded into the selected robust block acquired by spatial frequency criteria. The spatial frequency criteria improve the imperceptibility of the watermarked image. The benefit of block- based techniques is to attend the every block separately, which enhance the visual quality and robustness. This scheme has more embedding capacity and a little deterioration in the image due to shift invariant discrete wavelet transform (SIDWT) and singular value decomposition (SVD) properties. The future scope of this presented method is that to make it adaptive by using numerous optimization techniques. Experimental issues depicts that the presented scheme is tolerate the numerous image processing and noise attacks such as rotation, cropping, resizing, compression, Gaussian noise, salt and pepper noise, speckle noise and also perform better in terms of imperceptibility, robustness, capacity then previous presented scheme.

\section{REFERENCES}

1. I. Cox, M. Miller, J. Bloom, J. Fridrich, and T. Kalker, "Digital Watermarking and Steganography, 2nd Ed. (The Morgan Kaufmann Series in Multimedia Information and Systems)," pp. 28, 2007.

2. J. R. Hernandez, M. Amado, and F. Perez-Gonzalez, "DCT-domain Watermarking Techniques for Still Images: Detector Performance Analysis and a New Structure," IEEE Transaction Image Process, vol. 9, no. 1, pp. 55-68, 2000.

3. E. Hussein and M. A. Belal, "Digital Watermarking Techniques , Applications and Attacks Applied to Digital Media: A Survey," International Journal of Engineering Research \& Technology, vol. 1, no. 7, pp. 1-8, 2012.

4. Matt L. Miller, I.J Cox, J.P.M.G Linnartz, and T. Kalker, "A Review of Watermarking Principles and Practices, "Digital Signal Processing for Multimedia Systems, pp. 461-485, 1999.

5. C.H. Chou and K.C. Liu, "Robust and transparent watermarking scheme for colour images," IET Image Process., vol. 3, no. 4, pp. 228-242, 2009.

6. C. H. Huang and J. L. Wu, "Fidelity-guaranteed robustness enhancement of blind-detection watermarking schemes," Information Sciences, vol. 179, no. 6, pp. 791-808, 2009

7. R. K. Megalingam, M. M. Nair, R. Srikumar, V. K. Balasubramanian, and V. S. V Sarma, "Performance Comparison of Novel, Robust Spatial Domain Digital Image Watermarking with the Conventional Frequency Domain Watermarking Techniques," 2010 Int. Conf. Signal Acquis. Process (ICSAP), pp. 349-353, 2010.

8. G Bhatnagar, B.Raman "A new robust reference watermarking scheme based on DWT-SVD," Computer Standards \& Interfaces, 2009.

9. S. Gaur and V. K. Srivastava, "A Robust and Secure Block-SVD based Embedding of Encrypted Watermark in Digital Images using RDWT," International Journal of Security and its application, vol. 11, no. 1, pp. 257-270, 2017.

10. A. Al-Haj, "Combined DWT-DCT Digital Image Watermarking," J. Computer Science., vol. 3, no. 9, pp. 740-746, 2007.

11. N. M. Makbol and B. E. Khoo, "Robust blind image watermarking scheme based on Redundant Discrete Wavelet Transform and Singular Value Decomposition," Int. J. Electron. Communication (AEÜ ), vol. 67, pp. 102-112, 2013. 
12. C.C. Lai and C.C. Tsai, "Digital Image Watermarking Using Discrete Wavelet Transform and Singular Value Decomposition," IEEE Transaction Instrumentation Measurement, vol. 59, no. 11, pp. 3060-3063, 2010.

13. S. Gaur and V. Srivastava, "Robust Embedding of Improved Arnold Transformed Watermark in Digital Images using RDWT - SVD," in 2016 Forth International Conference on Parallel, Distributed and Grid Computing(PDGC), pp. 3-8, 2016.

14. A. Basso, F. Bergadano, D. Cavagnino, V. Pomponiu, and A. Vernone, "A Novel Block-based Watermarking Scheme Using the SVD Transform," Algorithms, vol. 2, no. 1, pp. 46-75, 2009.

15. R. Eko Caraka, Suparti, B. Warsito, and H. Yasin, "The Shift Invariant Discrete Wavelet Transform (SIDWT) with Inflation Time Series Application," Journal of Mathematics Research, vol. 8, no. 4, pp. 14-20, 2016.

16. O. Rockinger, "Image Sequence Fusion Using a Shift-Invariant Wavelet Transform," in Proceedings of International Conference on Image Processing, vol. 3, pp. 288-291, 1997.

17. C. E. Rezzy and Y. Hasbi, "Pemodelan Tinggi Pasang Air Laut Di Kota Semarang Dengan Menggunakan Maximal Overlap Discrete Wavelet Transform (MODWT)," J. Meteorol. Klimatologi dan Geofis., vol. 2, no. 2, pp. 1-30, 2015.

18. O. Renaud, J. L. Starck, and F. Murtagh, "Prediction Based on a Multiscale Decomposition," Int. J. Wavelets Multiresolution Inf. Processing, vol. 1, pp. 217-232, 2003.

19. S. Mallat, "A Theory for Multiresolution Signal Decomposition: The wavelet Representation," IEEE Transaction Pattern Analysis Machine Intelligence, vol. 11, no. 7, pp. 674-693, 1989.

20. R. A. Ghazy, A. M. Abbas, N. Al-zubi, E. S. Hassan, N. A. El, M. M. Hadhoud, M. I. Dessouky, S. A. Alshebeili, F. E. A. El-samie, "Block-based SVD image watermarking in spatial and transform domains, International Journal of Electronics " vol. 7217, no 20. September, 2015.

21. L. Cao, L. Jin, H. Tao, G. Li, Z. Zhuang, and Y. Zhang, "Multi-Focus Image Fusion Based on Spatial Frequency in Discrete Cosine Transform Domain," IEEE Signal Process. Letter, vol. 22, no. 2, pp. 220-224, 2015.

22. N. M. Makbol,B. E. Khoo and T. H. Rassem, "Block-based discrete wavelet transform-singular value decomposition image watermarking scheme using human visual system characteristics," IET Image Process., vol. 10 , no. 1 , pp. 34-52, 2016.

23. D. Kundur and D. Hatzinakos, "Toward Robust Logo Watermarking Using Multiresolution Image Fusion Principles," IEEE Transaction Multimedia, vol. 6, no. 1, pp. 185-198, 2004.

24. A. A. Reddy and B. N. Chatterji, "A new wavelet based logo-watermarking scheme," Pattern Recognition Letter, vol. 26, no. 7, pp. 1019-1027, 2005.

25. G. Bhatnagar, Q. M. Jonathan Wu, and B. Raman, "Robust gray-scale logo watermarking in wavelet domain," Computers and Electrical Engineering, vol. 38, no. 5, pp. 1164-1176, 2012.

26. C.H. Chen, Y.L. Tang, and W.S. Hsieh, "An Image Authentication and Recovery Method Using Optimal Selection of Block Types," 2014 IEEE Int. Symp. Multimedia, pp. 151-154, 2014.

27. R.Franklin.V, "Entropy based Robust Watermarking Scheme using Hadamard Transformation Technique," Int. J. Computer. Application, vol. 12, no. 9, pp. 14-21, 2011.

28. S. Li, J. T. Kwok, and Y. Wang, "Combination of images with diverse ocuses using the spatial frequency," Information Fusion, vol. 2, no. 3, pp. 169-176, 2001.

29. W. Xin, Y. L. Wei, and L. Fu, "A New Multi-Source Image Sequence Fusion Algorithm Based on SIDWT," 7th International Conference on Image and Graphics, (ICIG), pp. 568-571, 2013.

30. R. Liu, T. Tan, and S. Member, "An SVD-Based Watermarking Scheme for Protecting," vol. 4, no. 1, pp. 121-128, 2002 